\title{
Rubber plunger surface texturing for friction reduction in medical syringes
}

\author{
Haytam KASEM $^{1,2, *}$, Harel SHRIKI ${ }^{1}$, Lihi GANON ${ }^{1}$, Michael MIZRAHI ${ }^{3}$, Kareem ABD-RBO ${ }^{1,3}$, Abraham J. DOMB ${ }^{3}$ \\ ${ }^{1}$ Department of Mechanical Engineering, Azrieli College of Engineering, Jerusalem 9103501, Israel \\ ${ }^{2}$ Tribology Laboratory, Department of Mechanical Engineering, Technion, Haifa 32000, Israel \\ ${ }^{3}$ School of Pharmacy, Faculty of Medicine, the Hebrew University, Jerusalem 9103501, Israel
}

Received: 16 November 2017 / Revised: 27 March 2018 / Accepted: 21 June 2018

C The author(s) 2018. This article is published with open access at Springerlink.com

\begin{abstract}
Friction is a genuine issue in the use of many medical devices involving rubbery materials such as plungers in medical syringes. This paper presents a new direction for the reduction of friction in medical syringes based on surface texturing of the rubber plunger. The specimens were prepared by casting poly(vinylsiloxane) (PVS) rubber into a pre-fabricated negative template obtained by 3D printing. Friction tests were performed on a home-made test-rig. It was clearly shown that friction resistance can be considerably manipulated when using textured plungers.
\end{abstract}

Keywords: syringe; rubber; plunger; surface texturing; friction; medical syringes

\section{Introduction}

Low-friction combined with smooth movement is vital characteristic for drug-delivery devices particularly in medical syringes. Current materials and design, however, do not optimize these features. Indeed, many medical syringes use a rubber plunger for adequate sealing and compliance. In addition, it has been well established that the laws of metallic friction do not apply to rubber, since contacting asperities are primarily in the plastic loading range in the friction of metallic materials [1, 2]. In contrast, rubber asperities in contact remain generally in the elastic range [3]. Thus, appropriate friction mechanisms should be considered when investigating friction of rubber materials.

Relevant findings of many research projects on the friction of rubber lead to the current acceptance of three distinct friction mechanisms that develop, when rubber slides against harder counterface: adhesion [4], bulk deformation and hysteresis [5], and wear [6]. An early study published by Roth et al. indicates that the rubber coefficient of friction decreases as the applied normal load increases [7]. Another study published by Schallamach reports that, analogously to metals, the friction force generated by rubber is proportional to the real contact area of asperities contacting the antagonist surface [8].

It has also been reported that at higher speeds the compressed rubber surface in front of the asperity undergoes a buckling that produces detachment waves known as Schallamach waves [9]. In the light of the above, the complexity of rubber friction mechanisms indicates that friction is a practical issue for many uses of medical devices involving rubber, such as syringes, injection pens, and auto-injectors. For instance, a toohigh friction value between plunger and barrel in a medical syringe may influence the delivery of a drug, resulting in the patient receiving a spurt of the drug or a smaller than expected dose.

Known solutions to the problem often deal with plunger design. These solutions are generally protected by patents $[10,11]$. However, no attempts based on tribological considerations have yet been reported to resolve the problem of reducing friction between rubber

* Corresponding author: Haytam KASEM, E-mail: haytamka@jce.ac.il 
plunger and barrel in medical syringes.

Rubber surface texturing was considered for increasing friction in a wet contact condition. Kown et al. studied the friction of a micro-patterned elastomer in an attempt to enhance adhesion on a small intestinal surface for the development of friction-based clamping mechanisms for robotic endoscopic microcapsules [12]. Similarly, Tsipenyuk and Varenberg used rubber with a biomimetic hexagonal textured surface to increase friction against lubricated skin for shaving applications [13].

Even though rubber surface texturing has already been taken into account for increasing friction in a wet condition, it can be considered for decreasing friction in a wet condition like in medical syringes, as well. Thus, the present work aims at investigating, for the first time, the possibility of decreasing friction between rubber plunger and barrel in medical syringes by introducing surface texturing in the plunger. The concept is based on using a micro-surface structure in the form of micro-dimples obtained by casting rubber into a pre-fabricated negative template. The surface texturing in the form of micro dimples has been developed during the recent decades and was proved as an effective technology for reducing friction between mechanical components undergoing relative motion under lubricated condition [14]. It consists of the application of texturing to one of the two mating surfaces under lubricated contact. Due to relative motion, a hydrodynamic pressure is produced tending to separate the mating surfaces, thus reducing the friction between them. Indeed, each micro-dimple acts as a micro-hydrodynamic bearing to enhance hydrodynamic lubrication. The load carrying capacity provided by each micro-dimple is due to an asymmetric hydrodynamic pressure distribution over the dimple [15]. While this technology was essentially developed for hard materials such as sealing and cylinder liners $[16,17]$, in which both of the mating surfaces were rigid. Surface texturing, however, can also be effective in tribological contacts involving mating surfaces of which one is soft (Young's modulus around $10 \mathrm{MPa}$ ) $[18,19]$. In this case, the soft material may be deformed under the influence of the hydrodynamic pressure and viscous shear. Such cases are known as soft elastohydrodynamic lubrication (SEHL). Typical SEHL applications can be found in elastomeric seals [20-23], e.g., elastomer bearings [24], and metering size press [25].

In the present study we intend to apply this concept to textured rubber plungers (micro-dimples on the surface) and investigate by experiments the impact on the dynamic friction. To this end, three plungers having different textured surfaces but made of the same poly(vinylsiloxane) (PVS) rubber material have been prepared by casting. They were tested in medical syringes with three different fluids: water, alcohol (ethanol) and glycerol. Friction tests were performed at different sliding velocities.

\section{Experimental}

\subsection{Materials}

Poly(vinylsiloxane) (PVS) was obtained from Coltène Whaledent AG, Altstätten, Switzerland. This material has been used for the manufacturing of bionic textured surfaces for enhanced friction and adhesion [26, 27].

\subsection{Smooth and textured plungers}

The purpose of the present work is to investigate experimentally the possible positive effect of plunger surface texturing in reducing dynamic friction. Thus, we compare the frictional behavior of smooth and textured plungers. According to a theoretical model [28], the optimum dimples size of textured surface in rigid sealing is around 100 microns in diameter and 10 microns depth. This model was also validated experimentally [29]. Based on these data and taking into consideration the fact that in our case plungers are made from soft material (Young's modulus around $3 \mathrm{MPa}$ ) that is largely deformable, we chose to texture the plunger with dimples having 200 microns diameter and 20 microns depth. Textured and smooth plungers were prepared from the same PVS material and used to slide inside commercial medical syringes $(5 \mathrm{~mL}$, inner diameter $12.5 \mathrm{~mm}$ ). Of the three plungers one was completely smooth (used as reference), one textured with small dimple depth (20 microns, diameter 200 microns) and the $3^{\text {rd }}$ also textured with large dimple depth (50 microns, but the same diameter 200 microns) in order to study the possible influence of the depth. Smooth and textured plungers were prepared at the 
JCE's Solid \& Tribology Laboratory on three succeeding steps:

- At first, flat negative templates were prepared by 3D printing (3D Printing Center, The Hebrew University, Jerusalem, Israel) from high definition acrylate material. The 3D printing method was used for its good resolution and accuracy to achieve rapidly and easily complicated features as well as for its production time and cost effectiveness in possible future mass production. The quality of the obtained negative template was evaluated by 3D optical profilometer (Wyko NT1100, Veeco, Tucson, AZ, USA).

- Then, the negative templates were used to cast $1 \mathrm{~mm}$ thick strips textured on the front side (dimples) and smooth on the backside. These strips were casted from PVS using Young's modulus around $3 \mathrm{MPa}$ [30]. The cast thickness was controlled by using spacers of $1 \mathrm{~mm}$ height between the negative template and a smooth covering flat surface (see illustrations in Figs. 1(a)-1(c)).

- In the third step the obtained textured strip was cut to the right size of $5.9 \mathrm{~mm} \times 40.9 \mathrm{~mm}$ and rolled inside a master cylinder prepared by 3D printing, specifically to this end with an inner diameter of $12.9 \mathrm{~mm}$ (see illustration in Fig. 2). It is important to note that the inner diameter of the master cylinder was calculated based on the free state of the plunger (outside barrel) in medical syringes. This diameter, slightly larger than the inner diameter of the barrel (12.5 mm), guarantees a constant tolerance required for adequate contact pressure and sealing. The rolled strip fitted the master cylinder in one of two ways: (1) the textured side contacts the inner wall of the master cylinder to obtain textured plunger, or (2) the smooth back side contacts the wall of the master cylinder to obtain a smooth plunger. Then the tip of the piston was inserted inside the rolled textured strip, and PVS was used to fill the empty space between the rolled film and the tip. Once PVS was completely polymerized, the ready-to-use plunger was inserted into a new barrel (syringe).

- The specimens obtained (smooth and textured plungers) were imaged in an FEI Quanta 200 environmental SEM (FEI Co., Brno, Czech Republic) to check their quality (see example of textured plunger, dimples height 50 microns, in Fig. 3).
(a) Negative template
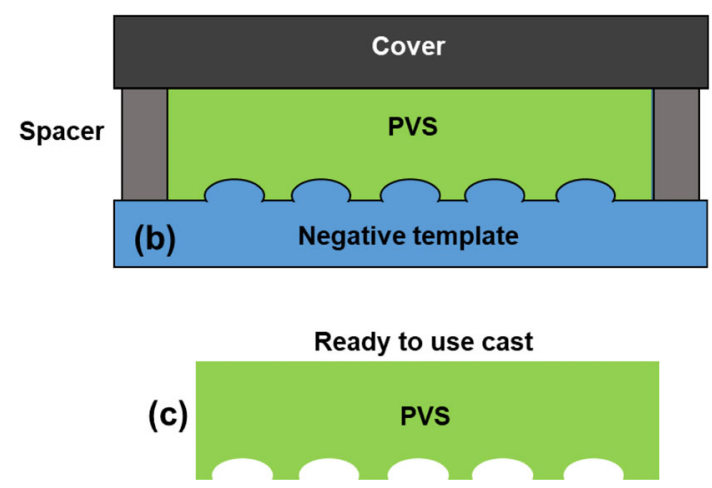

Fig. 1 Schematic illustration of casting process.

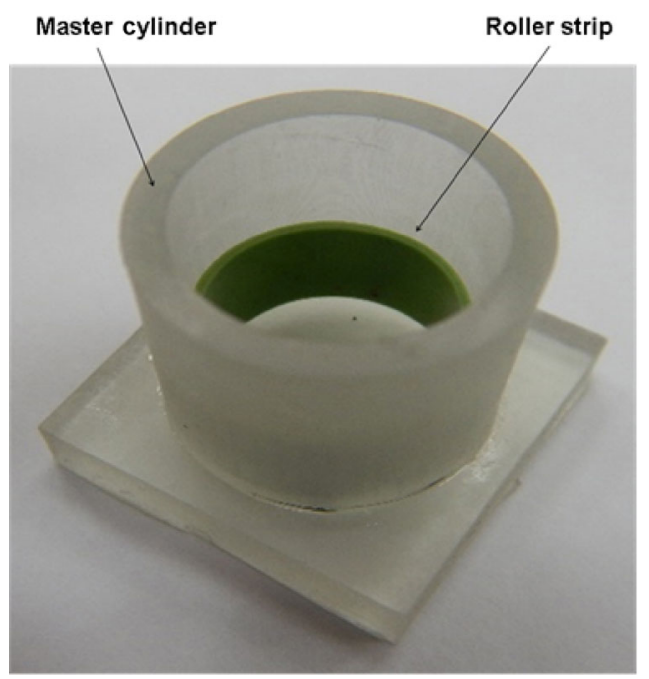

Fig. 2 Illustration of cast process to obtain plunger shape using textured strips.

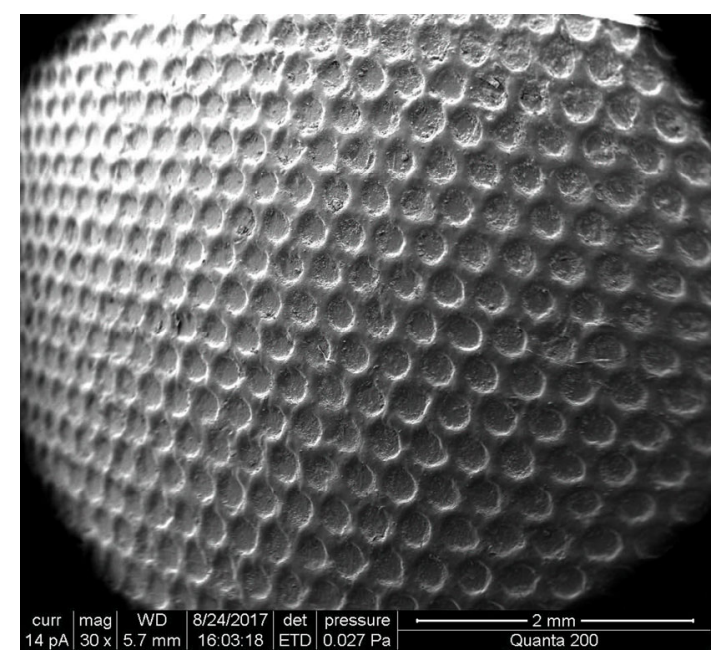

Fig. 3 SEM images of final plunger with textured surface (microdimples, height 50 microns). 


\subsection{Test apparatus description}

The friction tests were performed using a home-made test-rig that was specifically designed at the Solid \& Tribology Laboratory, the Jerusalem College of Engineering (JCE) to evaluate frictional performances between plunger and barrel in medical syringes. The device was constructed on the basis of a one-passvertical displacement consisting of driving and measuring units. Figure 4 presents a general view of the experimental test-rig incorporating the different elements used for controlled vertical displacement and measuring force. A medical syringe $\{1\}$ whose rubber plunger underwent surface texture modification was held on a rigid holder $\{2\}$. The plunger's tip head was pressed by a motorized linear actuator (A-LAR300APC01 , US) $\{3\}$ at a constant velocity. A recipient $\{5\}$ was placed below the syringe to collect the injected liquid. The friction force resisting the sliding of the plunger inside the barrel was recorded by the measurement unit consisting of an accurate load cell FUTEK (FSH00098, US) $\{4\}$. The measurements were sampled

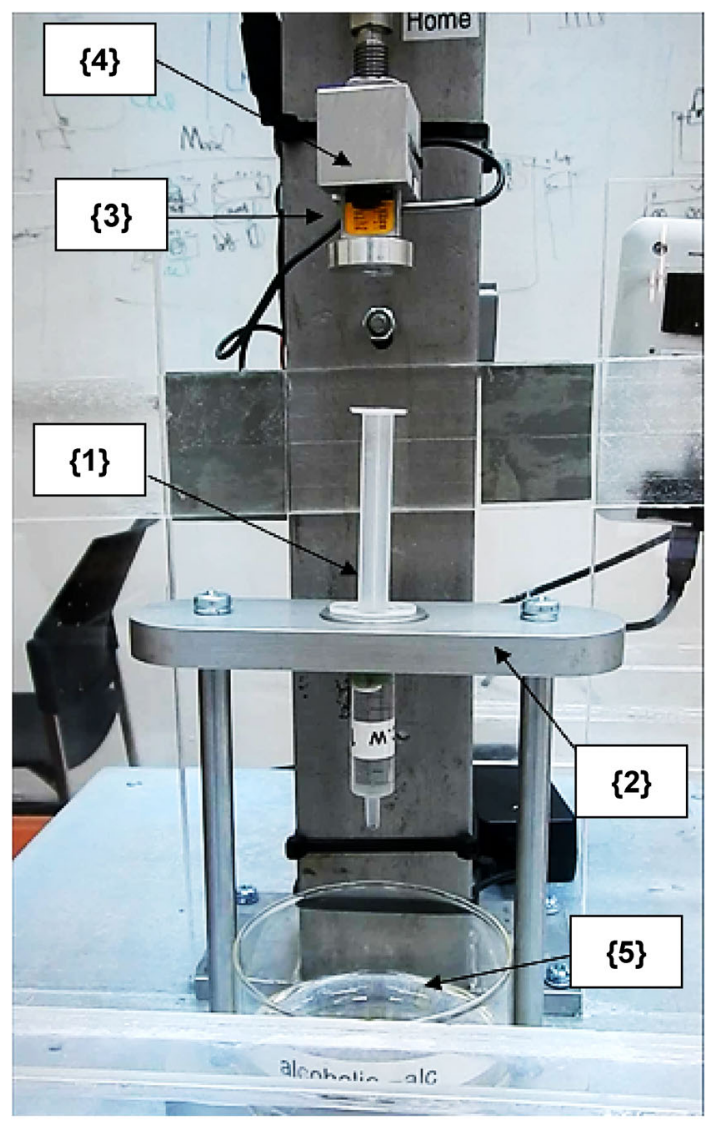

Fig. 4 General view of the used homemade experimental test-rig. with a multifunctional data acquisition board LabPC-1200 (National Instruments Co., Austin, Texas, USA) and processed using a LabVIEW software package (National Instruments Co., Austin, Texas, USA).

\subsection{Test procedure}

The plunger is inserted inside the barrel, then the syringe is filled with the desired liquid and positioned in the rigid holder. All tests begin by pre-touch, during which the head of the vertical actuator is brought into contact with the upper part of the sliding piston of the syringe at a small sliding velocity of $2 \mathrm{~mm} / \mathrm{s}$, until a certain pre-load of $0.5 \mathrm{~N}$ is reached, then the motion is stopped. The value of the pre-load was determined by preliminary tests with the different liquids to set the smaller value of the pre-load that guarantees identical initial conditions for the different tests (pre-position of the rubber plunger and eliminating possible clearance leading to reproducible and stable frictional behavior during the posterior test). Once pre-touch is accomplished, the tests are performed under a predefined constant sliding velocity and a total stroke distance. All tests are performed at a room temperature of $21-22{ }^{\circ} \mathrm{C}$ and a relative humidity ranging between $45 \%-50 \%$.

\section{Results and discussion}

Each plunger was tested with three different liquids, i.e., water, glycerol and alcohol (ethanol). In Table 1 the characteristics of the different tested liquids are reported. The tests were conducted under three characteristic sliding velocities $V$, i.e., 2.5, 5, and $10 \mathrm{~mm} / \mathrm{s}$. Figure 5 presents a graph showing the typical variation of friction force resisting the motion of the plunger inside the barrel during a test conducted with glycerol at $2.5 \mathrm{~mm} / \mathrm{s}$ for the three different plungers (smooth, textured $20 \mu \mathrm{m}$ height, textured $50 \mu \mathrm{m}$ height). This behavior can be divided into three characteristic stages: Stage $\{1\}$ relates to friction increase during

Table 1 Characteristics of the tested liquids at $20{ }^{\circ} \mathrm{C}$.

\begin{tabular}{ccc}
\hline & Density, $\rho\left(\mathrm{kg} / \mathrm{m}^{3}\right)$ & Viscosity, $\mu\left(\mathrm{N} \cdot \mathrm{s} / \mathrm{m}^{2}\right)$ \\
\hline Water & 999.8 & 1.002 \\
Alcohol & 786.5 & 0.0011 \\
Glycerin & 1259 & 0.95 \\
\hline
\end{tabular}




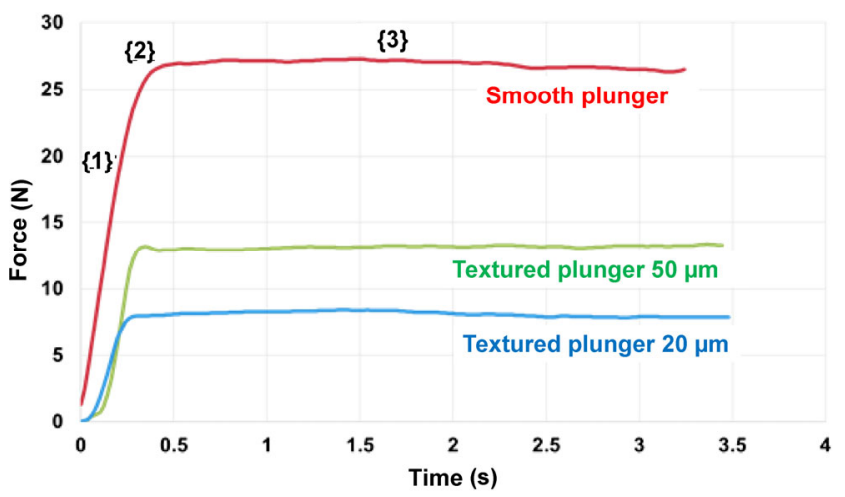

Fig. 5 Typical behavior of friction force resisting the motion of the plunger inside the barrel during a test realized with glycerol under sliding velocity of $2.5 \mathrm{~mm} / \mathrm{s}$.

pre-sliding and elastic deformation of the plunger; Stage $\{2\}$ designates the maximum static friction force reached at sliding inception; and finally, Stage $\{3\}$ corresponds to the stabilization of the dynamic friction force during full sliding of the plunger. It is important to note that for all tests we verified that no liquid leakage occurred between the plunger and the barrel of the syringe. In this study, the average stabilized friction force during sliding (Stage $\{3\}$ ) was recorded for each test. The average resistance friction force $F_{\mathrm{R}}$ and errors bars (obtained from 4 repetitions) are computed for the three plungers tested at the different sliding velocity $V$ when considered separately and presented in Figs. 6(a)-6(c).

It should be clarified that the measured resistance force $F_{\mathrm{R}}$ is composed of two main sources, namely the friction resistance $F_{\mathrm{F}}$ between the plunger and the inner wall of the barrel; and, the liquid inner shearing forces $F_{\mathrm{S}}$, which depends on its viscosity (see Eq. (1)).

$$
F_{\mathrm{R}}=F_{\mathrm{F}}+F_{\mathrm{S}}
$$

Although the measured friction force is composed of two different source, when considering tests conducted with the same liquid at the same sliding velocity, the liquid shearing force $F_{\mathrm{S}}$ is assumed to remain constant. Besides taking into consideration that all three plungers are only differentiated by surface texture, all other parameters such as material and macroscopic dimensions were kept constant. It is, therefore, valid to assume that in this case the variation of the resistance force $F_{R}$ can be related to the unique change in the friction force $F_{\mathrm{F}}$ and related to plunger surface texturing.

Three main observations can be made from these results (Fig. 6):

- The resistance friction force $F_{\mathrm{R}}$ is always higher in the case of a smooth plunger regardless of the liquid used and/or sliding velocity. This finding highlights the fact that using a textured plunger reduces the resistance friction force. To explain these results, we should consider the relative motion of two surfaces
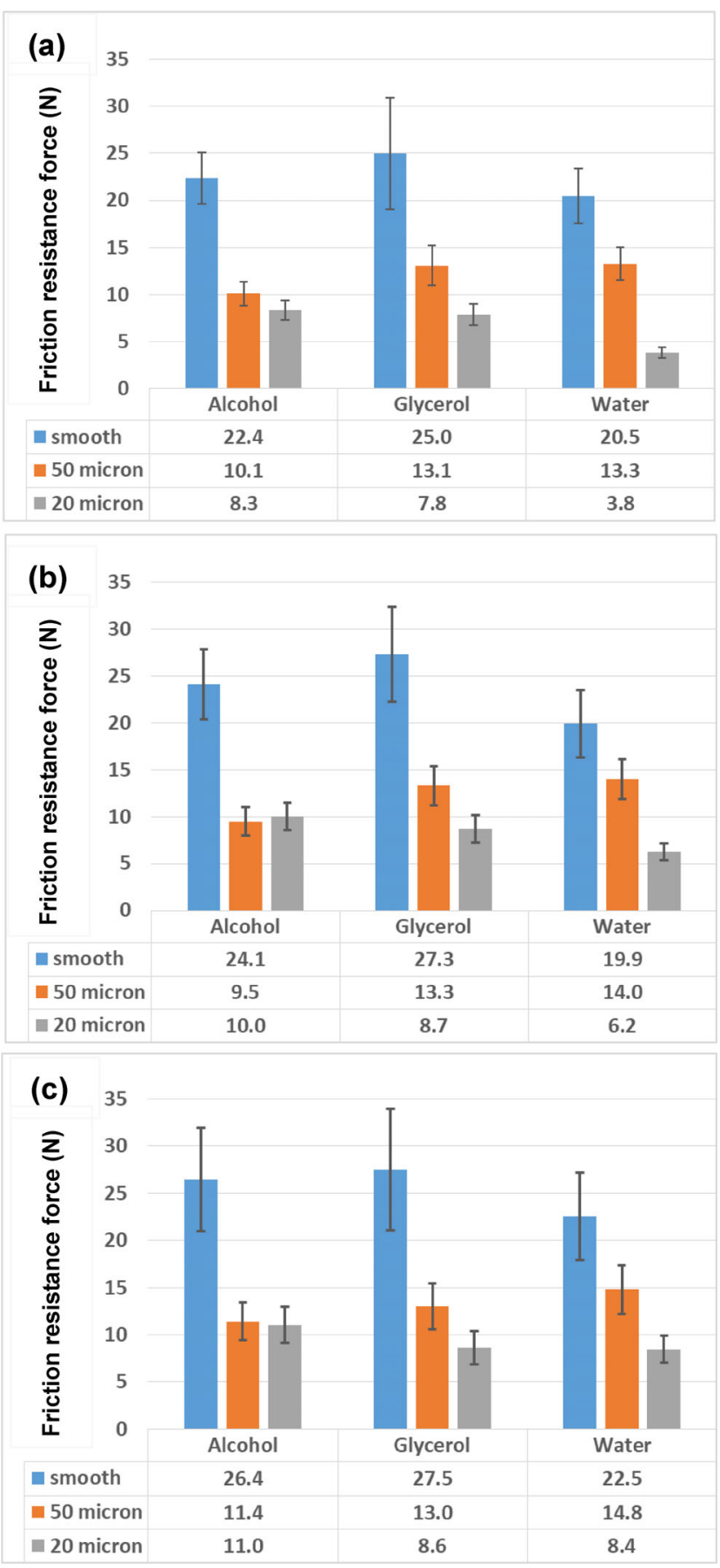

Fig. 6 Average friction resistance force $F_{\mathrm{R}}$ and error bars for the three plungers at sliding velocity (a) 2.5 , (b) 5 , and (c) $10 \mathrm{~mm} / \mathrm{s}$. 
against each other with the presence of a lubricant that flows through a converging wedge forming a hydrodynamic fluid film between them [31]. The variation of lubricant pressure in a converging wedge is described by the Reynolds equation that allows determining the pressure distribution and load capacity $[32,33]$. A full film lubrication regime can be achieved, if the thickness of the lubricant film is large enough, compared to the average roughness of the surface, to entirely separate the two mating surfaces [34]. To illustrate the positive effect of the surface texturing (micro spherical dimples as considered in the present work) in increasing the hydrodynamic pressure between two sliding surfaces, which allows to separate them and reduce thus the friction, it is necessary to examine the hydrodynamic effect that occurs at the converging and diverging zones of an individual micro dimple (see schematic illustration in Fig. 7). Then, the plurality of micro dimples can be considered as a set of micro bearings. The pressure within the lubricant film increases in the converging area and decreases in the diverging area. Therefore, the positive effect of surface texturing can be obtained only if the increase of pressure at the converging zone of the micro dimples is higher than its decrease at the diverging zones. This can explain why, in the case of a smooth plunger (without dimple), the friction force is higher than those obtained with the textured surfaces.

- By comparing the frictional performances of the two textured plungers, it can clearly be remarked that the smaller texture height (dimple height of 20 micros) generates smaller resistance force. It is important to recall that the two textured plungers have the same dimple diameter and the same aspect ratio. Thus, the real contact surface between the textured plunger and the inner wall of the barrel (syringe) is equal, as well. Therefore, the difference in terms of the resistance force developed by the two textured plungers can only be related to the features of the dimples. Given that in the case of high dimple height (50 micros) the size of the converging wedge is higher, it can be assumed that the pressure increase within the lubricant film is smaller compared to the small dimple height (20 micros). This difference can explain why the friction force is smaller in the case of a textured plunger with small dimple height
(20 micros). This also suggests the existence of optimized texturing height giving enhanced performance. This point will be considered in future work.

- While the friction resistance force decreases notably between the large height and small height dimples of the textured plunger when tested with water and glycerol, conversely in the case of alcohol the variation is too small or even non-existent. Obviously, this can be related to the small viscosity of the alcohol (ethanol) compared to those of the water and glycerol (see Table 1), which do not generate sufficient pressure within the lubricant film under the considered operational conditions.

\section{Conclusion}

The influence of rubber plunger surface texturing on the resistance friction force in medical syringes was investigated experimentally. To this end, a home-made experimental test-rig was specifically designed at the Solid \& Tribology Laboratory in JCE to evaluate frictional performance between plunger and barrel in medical syringes. The device was constructed on the basis of a one-pass-vertical displacement. The plungers were prepared from PVS. Textured surfaces were obtained by casting PVS into a negative template prepared by 3D printing. Different injected liquids including water, alcohol and glycerol were used to

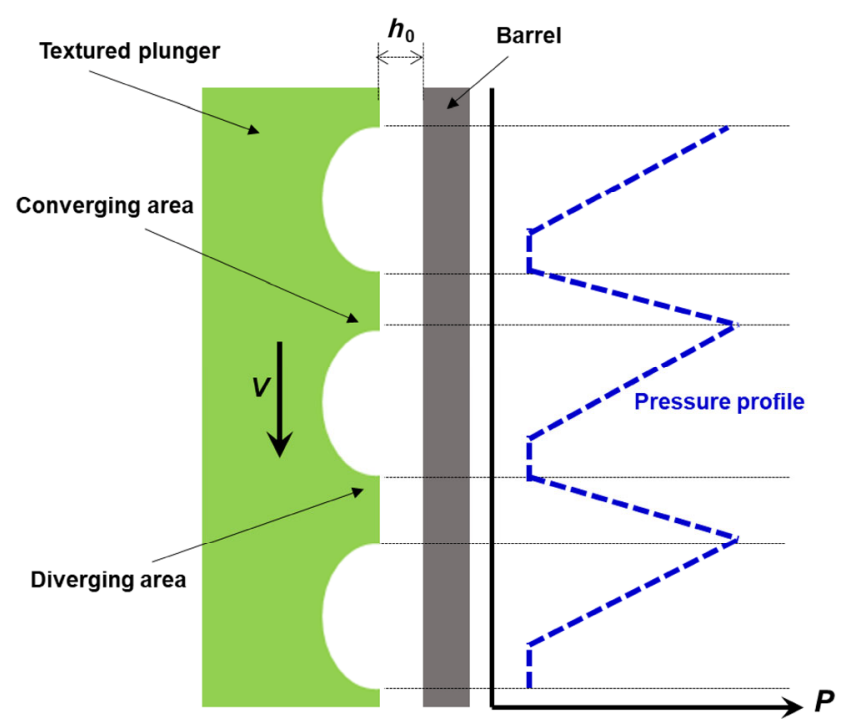

Fig. 7 Typical pressure distribution in plunger with spherical micro textured surface. 
conduct friction tests. The tests were performed at three different sliding velocities, i.e., $2.5,5$ and $10 \mathrm{~mm} / \mathrm{s}$. The following observations were made:

- The resistance friction force decreases notably with the textured plungers, regardless of the liquid used and/or sliding velocity.

- The smaller texture (dimples height of 20 micros) generates a smaller resistance force, suggesting the existence of an optimized design for enhanced frictional performances.

- In contrast to water and glycerol, alcohol decreases the friction resistance force between large and small texturing (dimples height). The friction is too small or even non-existent. This can be related to the small viscosity of the alcohol (ethanol), which does not allow the generation of sufficient high pressure within the lubricant film at the converging zones of the micro dimples.

\section{Acknowledgements}

The negative templates for the casting of textured plungers were prepared at the Center for Functional and 3D Printing, at the Hebrew University of Jerusalem. The experimental test-rig was funded by Maof Fellowships, the Council for Higher Education of Israel (Dr. H. Kasem), and the MIA program the Ministry of Science, Technology \& Space (Prof. A. Domb).

Open Access: The articles published in this journal are distributed under the terms of the Creative Commons Attribution 4.0 International License (http:// creativecommons.org/licenses/by/4.0/), which permits unrestricted use, distribution, and reproduction in any medium, provided you give appropriate credit to the original author(s) and the source, provide a link to the Creative Commons license, and indicate if changes were made.

\section{References}

[1] Bowden F O, Tabor D. The Friction and Lubrication of Solids. 2nd ed. Oxford (UK): Clarendon Press, 2001.

[2] Rabinowitz E. Friction and Wear of Materials. 2nd ed. New York (USA): John Wiley \& Sons, 1995.

[3] Smith R H. Analyzing Friction in the Design of Rubber Products and Their Paired Surfaces. CRC Press, Taylor \&
Francis Group NW, Book Number 13: 978-0-8493-8136-2, 2008.

[4] Thirion P. Les coefficients d'adherence du caouchouc. (in French). Rev Gen Caoutch 23: 101 (1946)

[5] Grosch K A. The relation between the friction and viscoelastic properties of rubber. Proc Roy Soc London Ser A: Math Phys Sci 274(1356): 21-39 (1963)

[6] Muhr A H, Roberts A D. Rubber abrasion and wear. Wear 158(1-2): 213-228 (1992)

[7] Roth F L, Driscoll R L, Holt W L. Frictional proprieties of rubber. J Res Nat Bur Stds 28: 439 (1942)

[8] Schallamach. How does rubber slide? Wear 17(4): 301-312 (1971)

[9] Barquins M. Sliding friction of rubber and Schallamach waves - A review. Mater Sci Eng 73: 45-63 (1985)

[10] Gross J R. Low friction syringe. U.S. Patent 5397 313A, Mar. 1995.

[11] Merry J D. Low friction syringe. U.S. Patent 4 26557A, May 1981.

[12] Kwon J, Cheung E, Park S, Sitti M. Friction enhancement via micro-patterned wet elastomer adhesives on small intestinal surfaces. Biomed Mater 1(4): 216-220 (2006)

[13] Tsipenyuk A, Varenberg M. Use of biomimetic hexagonal surface texture in friction against lubricated skin. J Roy Soc Interface 11(94): 20140113 (2014)

[14] Etsion I. State of the art in laser surface texturing. J Tribol 127(1): 248-253 (2005)

[15] Gachot C, Rosenkranz A, Hsu S M, Costa H L. A critical assessment of surface texturing for friction and wear improvement. Wear 372-373: 21-41 (2017)

[16] Etsion I, Kligerman Y, Halperin G. Analytical and experimental investigation of laser-textured mechanical seal faces. Trib Trans 42(3): 511-516 (1999)

[17] Etsion I, Halperin G, Ryk G. J Balkan Tribol Assoc 6: 72-77 (2000)

[18] Hadinata P C, Stephens L S. Soft elastohydrodynamic analysis of radial lip seals with deterministic microasperities on the shaft. J Tribol 129(4): 851-859 (2007)

[19] Shinkarenko A, Kligerman Y, Etsion I. The effect of surface texturing in soft elasto-hydrodynamic lubrication. Tribol Int 42(2): 284-292 (2009)

[20] Shi F H, Salant R F. Numerical study of a rotary lip seal with a quasi-random sealing surface. J Tribol 123(3): 517-524 (2001)

[21] Salant R F, Maser N, Yang B. Numerical model of a reciprocating hydraulic rod seal. J Tribol 129(1): 91-97 (2006)

[22] Nikas G K. Theoretical study of solid back-up rings for elastomeric seals in hydraulic actuators. Tribol Int 37(9): 689-699 (2004)

[23] Stupkiewicz S, Marciniszyn A. Elastohydrodynamic lubrication and finite configuration changes in reciprocating 
elastomeric seals. Tribol Int 42(5): 615-627 (2009)

[24] Hirani H, Verma M. Tribological study of elastomeric bearings for marine propeller shaft system. Tribol Int 42(2): 378-390 (2009)

[25] Bohan M F J, Lim C H, Korochkina T V, Claypole T C, Gethin D T, Roylance B J. An investigation of the hydrodynamic and mechanical behaviour of a soft nip rolling contact. Proc Instit Mechan Eng J: J Eng Tribol 211(1): 37-49 (1997)

[26] Kasem H, Tsipenyuk A, Varenberg M. Biomimetic wallshaped hierarchical microstructure for gecko-like attachment. Soft Matter 11(15): 2909-2915 (2015)

[27] Tuma J. U.S. Patent 20070063375 A1. 2007.

[28] Ronen A, Etsion I, Kligerman Y. Friction-reducing surfacetexturing in reciprocating automotive components. Tribol

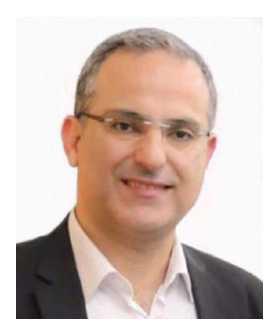

Haytam KASEM. He received his M.S. degree in mechanical engineering from the University of Haute Alsace in Mulhouse, France, in 2004. He received his $\mathrm{PhD}$ degree in tribology of composite materials from the University of Orleans,
Trans 44(3): 359-366 (2001)

[29] Ryk G, Kligerman Y, Etsion I. Experimental investigation of laser surface texturing for reciprocating automotive components. Tribol Trans 45(4): 444-449 (2002)

[30] Peressadko A, Gorb S N. When less is more: Experimental evidence for tenacity enhancement by division of contact area. $J$ Adhes 80(4): 247-261 (2004)

[31] Reynolds O. On the theory of lubrication and its applications. Proc R Soc Lond Ser A-Math Phys Eng Sci 40: 191-203 (1886)

[32] Stachowiak G W, Batchelor A W. Engineering Tribology. Boston (UK): Butterworth Heinemann, 2001.

[33] Williams J A. Engineering Tribology. Oxford (UK): Oxford University Press, 1994.

[34] Cameron A, Ettles C M M. Basic Lubrication Theory. Chichester: Ellis Horwood Ltd., 1983.

France, in 2008. He joined the Tribology Laboratory at Technion, Israel, in 2012 and the Azrieli College of Engineering in Jerusalem, Israel, in 2013. His current position is an assistant professor. His research areas cover the tribology of bionic microstructures, biotribology, mechano-chemical surface treatment and tribology of friction brakes. 